Title:

\title{
An optimal growth law for RNA composition and its partial implementation through ribosomal and tRNA gene locations in bacterial genomes
}

\section{Authors:}

Xiao-Pan Hu, Martin J. Lercher*

Xiao-Pan Hu, Institute for Computer Science and Department of Biology, Heinrich Heine University,

Martin J. Lercher, Institute for Computer Science and Department of Biology, Heinrich Heine University, D-40225 Düsseldorf, Germany; martin.lercher@hhu.de

* Correspondence to: martin.lercher@hhu.de 


\section{Abstract}

The distribution of cellular resources across bacterial proteins has been quantified through phenomenological growth laws. Here, we describe a complementary bacterial growth law for RNA composition, emerging from optimal cellular resource allocation into ribosomes and ternary complexes. The predicted decline of the tRNA/rRNA ratio with growth rate agrees quantitatively with experimental data. Its regulation appears to be implemented in part through chromosomal localization, as rRNA genes are typically closer to the origin of replication than tRNA genes and thus have increasingly higher gene dosage at faster growth. At the highest growth rates in E. coli, the tRNA/rRNA ratio appears to be regulated entirely through this effect.

\section{Keywords}

RNA; ribosomal RNA; tRNA; resource allocation; bacterial growth laws; genomic location; gene expression

\section{Background}

The systematic change of the coarse-grained composition of bacterial proteomes with growth rate $[1,2]$ an apparently linear increase of the ribosomal protein fraction with growth rate $[1,3]$. These laws have been successfully applied to the prediction of a range of phenotypic observations [3,5-8]. Recently, it has been argued that they arise from an optimal balance between the cellular investment into catalytic proteins and their substrates [9].

35 In contrast to the proteome composition, the partitioning of bacterial RNA into messenger (mRNA), ribosomal (rRNA), and transfer (tRNA) RNA is often assumed to be growth rate-independent $[2,3,5,6,10,11]$. However, experimental evidence suggests that the tRNA/rRNA expression ratio decreases 
monotonically with growth rate [12-20], suggesting the existence of a bacterial growth law for RNA composition.

The regulatory implementation of bacterial growth laws is generally assumed to arise from a small number of major transcriptional regulators such as ppGpp [21,22] and cAMP [4,23]. However, growth-rate dependent transcriptional regulation could also be implemented through chromosomal gene positioning. Prokaryotic genes are non-randomly located on multiple levels [24-26], with highly expressed genes biased towards the origin of replication (oric) [27]. The latter observation is thought to facilitate high 45 expression levels at fast growth, where, due to the presence of partially replicated chromosomes, gene copy numbers depends on the distance to oriC (replication-associated gene dosage) [28-30]. Indeed, chromosome rearrangements that shift highly expressed genes from the origin to the terminus of replication reduce fitness [31-35].

rRNA forms the central part of the catalyst of peptide elongation, while tRNA forms the core of the substrate; together, they account for the bulk of cellular RNA [2]. Their cytosolic concentrations at different growth rates in $E$. coli are well described by an optimality assumption $[9,36,37]$. Moreover, chromosomal gene positions in E. coli are known to affect the expression of both tRNA and rRNA genes $[38,39]$; both types of genes are located closer to oriC in fast compared to slowly growing bacteria, with rRNA genes positioned closer to oriC than tRNA genes in most examined fast-growing bacteria [27]. can be described by a bacterial growth law that arises from optimal resource allocation and (ii) that this growth law is at least partially implemented through the relative chromosomal positioning of tRNA and rRNA genes. 


\section{Results and discussion}

In terms of dry mass allocation, translation is the most expensive process in fast-growing bacteria [2,40].

As evidenced by comparison of diverse data to a detailed biochemical model of translation, the allocation of cellular resources across components of the E. coli translation system minimizes their total dry mass concentration at a given protein production rate [37]. This result indicates that natural selection favored the parsimonious allocation of cellular resources to the translation machinery in E. coli. To generalize this optimization hypothesis to other species, we here analyze a coarse-grained translation model that only considers peptide elongation, where the active ribosome acts as an enzyme that converts ternary complexes (TC), consisting of elongation factor Tu (EF-Tu), GTP, and charged tRNA, into an elongating peptide chain following Michaelis-Menten kinetics $[5,41]$. In exponential, balanced growth at rate $\mu$ and cellular protein concentration $P$, the total rate of protein production is $v=\mu \cdot P$. We derived the optimal concentration ratio of TC (with molecular mass $m_{\mathrm{TC}}$ ) to ribosome $\left(R\right.$, with molecular mass $\left.m_{\mathrm{R}}\right)$ at this production rate by minimizing their combined mass concentration, $M_{\text {total }}=m_{\mathrm{TC}}[\mathrm{TC}]+m_{\mathrm{R}}[R]$ (Methods):

$$
\frac{[\mathrm{TC}]}{[R]}=\frac{a \cdot \sqrt{k_{\mathrm{cat}} \cdot K_{\mathrm{m}}}}{\sqrt{a \cdot \mu \cdot P}+\sqrt{k_{\mathrm{cat}} \cdot K_{\mathrm{m}}}} ;
$$

Here, $a=\frac{m_{\mathrm{R}}}{m_{\mathrm{TC}}}=33.1$ is the ratio of molecular weights of ribosome and TC; $k_{\text {cat }}$ is the turnover number of the ribosome; and $K_{\mathrm{m}}$ is the ribosome's Michaelis constant for TC, which is limited by diffusion [5].

75 For a given genome, $a, k_{\mathrm{cat}}$, and $K_{\mathrm{m}}$ can be treated as constants [5,6]. Moreover, the cellular protein mass concentration $P$ appears to be similar across most species [42] and shows only minor variations across growth rates in those bacteria where it has been tested $[7,43,44]$. Thus, equation (1) predicts that the $\mathrm{TC} /$ ribosome ratio is a monotonically decreasing function of the growth rate $\mu$. Since most cellular EF-Tu and tRNA are present in the form of TCs [5], hereafter, the TC concentration is assumed to be approximately equal to the concentration of EF-Tu and tRNA. 
To calculate the optimal TC/ribosome ratio in E. coli, we use the measured protein concentration $P$ [45], set the turnover number $k_{\text {cat }}$ to the maximal observed translation rate [2], and set the Michaelis constant $K_{\mathrm{m}}$ to its diffusion limit [5] (Methods; see also Ref. [37]). Fig. 1a compares the optimal predictions (red line) to experimental datasets that estimated the TC/ribosome ratio based on ratios of tRNA/rRNA [20,46,47], EF-Tu/rRNA [19], and EF-Tu/ribosomal proteins [45] (Additional file 1: Table S1). Consistent with the predictions, all experimental estimates of the TC/ribosome ratio are approximately two-fold higher at low compared to high growth rates. As the TC and ribosome constitute the two major
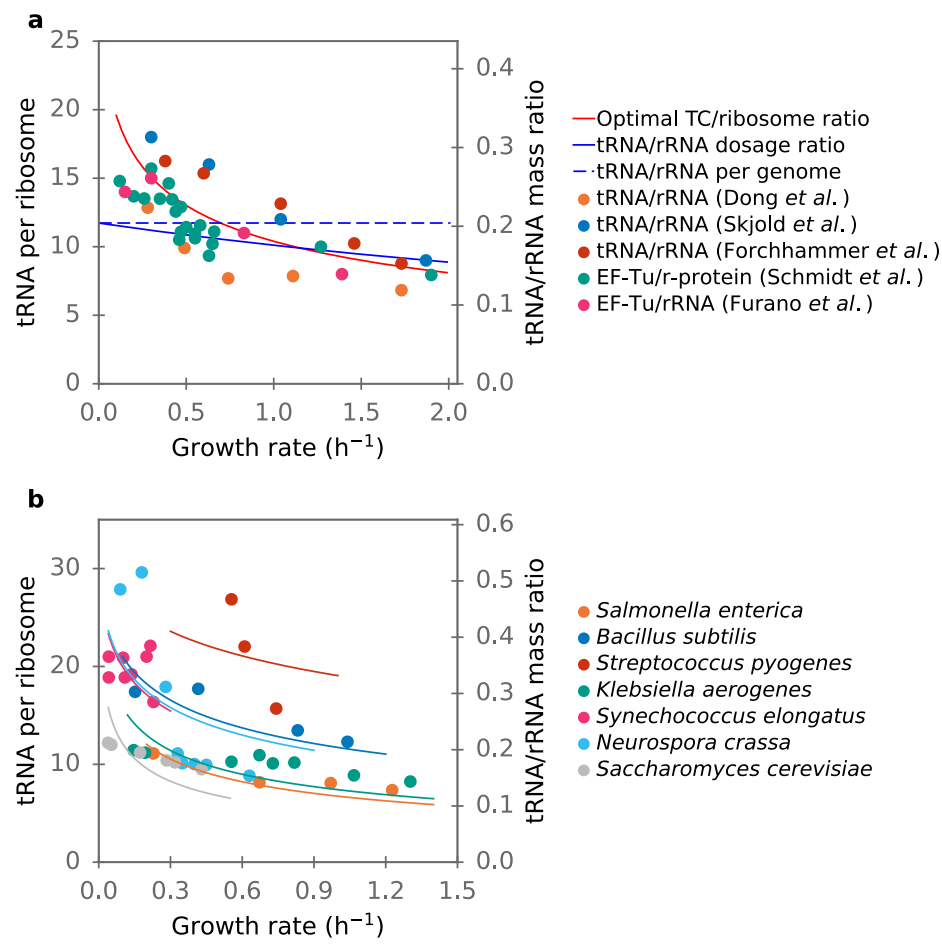

\section{Figure 1. The RNA composition growth law predicts decreasing TC/ribosome ratios with growth rate.}

(a) Different experimental estimates of TC/ribosome ratios in E. coli are consistent with the optimal ratio according to equation (1) (red line). The solid blue curve marks the replication-associated tRNA/rRNA dosage ratio based on chromosomal positions of tRNA and rRNA genes; the dashed blue line marks the expectation based on the tRNA/ribosome gene ratio per chromosome. (b) Experimental estimates of $\mathrm{TC} /$ ribosome ratios in different microbes are also largely consistent with the optimal ratio; for each dataset, we fitted equation (1) to the data by varying the single adjustable parameter $k$ (colored lines). 
components of cellular RNA [2], we conclude that the optimal TC/ribosome ratio according to equation (1) represents a bacterial growth law for RNA composition:

$$
\frac{M_{\mathrm{tRNA}}}{M_{\mathrm{rRNA}}}=r \cdot \frac{\sqrt{k_{\mathrm{cat}} \cdot K_{\mathrm{m}}}}{\sqrt{a \cdot \mu \cdot P}+\sqrt{k_{\mathrm{cat}} \cdot K_{\mathrm{m}}}},
$$

where $M_{\mathrm{tRNA}}$ and $M_{\mathrm{rRNA}}$ are the cellular mass of tRNA and rRNA, respectively, and $r=0.58$ is the ratio of the tRNA mass fraction of a TC and the rRNA mass fraction of the bacterial ribosome (Methods).

The approximate Michaelis-Menten form of the rate law for peptide elongation, on which the above equations are based, arises from the structure of the detailed elongation process [41]. As this process is shared by all living cells [41], we expect that the RNA composition growth law (2) holds also for other fastgrowing microbes (with $a=40.3$ and $r=0.59$ in eukaryotes). To test this hypothesis, we collected all available tRNA/rRNA ratios in microbes (Fig. 1b and Additional file 1: Table S2). Note that if protein concentration $P$ is indeed approximately constant across species [42], then equations (1) and (2) contain a single species-specific parameter, the geometric mean of $k_{\text {cat }}$ and $K_{\mathrm{m}}, k:=\sqrt{k_{\text {cat }} \cdot K_{\mathrm{m}}}$.

For six out of the seven datasets in Fig. 1, the tRNA/ribosome ratio decreases with increasing growth rate.

100 The only exception, the cyanobacterium Synechococcus elongatus, has a much smaller maximal growth rate $\left(\mu_{\max }=0.23 \mathrm{~h}^{-1}\right)$ than the other species, and its tRNA/rRNA ratio does not show a clear growth ratedependence [48]. It is conceivable that slow-growing species do not fully optimize their translation machinery composition, as a near-optimal constant TC/ribosome ratio may incur a lower fitness cost than a regulatory system for growth rate-dependent expression.

105 We define gene dosage as the DNA copy number of a given gene in one cell. Gene dosage can change permanently because of chromosomal duplications and deletions, but also transiently according to the progress of chromosome replication. The latter causes a gene position-dependent dosage effect, with genes closer to the origin of replication (oriC) having a higher average dosage than genes further away 
(equation (14)). Gene dosage effects are in particular known to affect the expression of rRNA [38] and

tRNA genes [39]. Moreover, a strong selection pressure towards optimal tRNA/ribosome ratios appears

to exist in fast-growing bacteria (Fig $\mathbf{1 b}$ ). We thus hypothesized that the relative genomic position of tRNA and rRNA genes may contribute to the implementation of the RNA composition growth law (Fig 2a). Here and below, for each genome, we summarize the multiple tRNA genes by averaging over their positions; we do the same for the rRNA genes.

115 In line with previous analyses $[27,49]$, we found that in fast-growing species (minimal doubling time $\leq 1 \mathrm{~h}$, i.e., $\mu_{\max } \geq 0.69 \mathrm{~h}^{-1}$ ), rRNA and tRNA genes are generally located in the vicinity of oriC, at relative positions $<0.5$ (Fig. 2 b,c, orange points; $\mu_{\max }$ from Ref. [49]). Moreover, we found that both rRNA and tRNA genes tend to be located ever closer to oriC with increasing $\mu_{\max }$ (Spearman's rank correlation coefficient between $\mu_{\max }$ and position ${ }_{\mathrm{rRNA}}: \rho=-0.59, P=9.2 \times 10^{-6}$; between $\mu_{\max }$ and position ${ }_{\mathrm{tRNA}}: \rho=-0.40$, $P=0.0047)$. In slow-growing species, rRNA genes still tend to be close to oriC (Fig. 2 b, blue points), while tRNA genes are distributed around the midpoint between oriC and the terminus (Fig. 2c, blue points). We found that rRNA genes are closer to oriC than tRNA genes in most slow-growing and in all but one fastgrowing bacteria (Fig. 2d). To analyze how this affects the tRNA/rRNA ratio, we used the model developed by Bremer and Churchward [30] for the dosage ratio of two genes at growth rate $\mu$,

$$
\frac{X_{i}}{X_{j}}=e^{C \cdot \mu \cdot\left(\text { position }_{j}-\text { position }_{i}\right)}
$$

125 here, $X_{i}$ is the dosage and position $i$ is the position of gene $i$, and $C$ is the time required to complete one round of chromosome replication (see Methods for details). The DNA replication rate varies between species but is approximately constant across conditions in a given species [50], making the gene dosage ratio a monotonous function of the growth rate [51,52]. For tRNA and rRNA with multiple genes, the overall dosage ratio can be described by a generalization of equation (3) (Methods, equation (16)). As rRNA gene positions tend to be smaller than tRNA positions (Fig. 2d), the tRNA/rRNA ratio that would be 
obtained if regulation was exclusively by gene dosage is a decreasing function of growth rate, in qualitative agreement with the optimality predictions from equation (2).

This finding supports our hypothesis that natural selection has fine-tuned the positions of tRNA and rRNA genes to match the RNA composition growth law for optimally efficient translation. This can best be seen for E. coli, for which all necessary parameters are available, allowing us to make quantitative predictions without adjustable parameters (Fig. 1a). Here, the tRNA/rRNA dosage ratio at higher growth rates

a
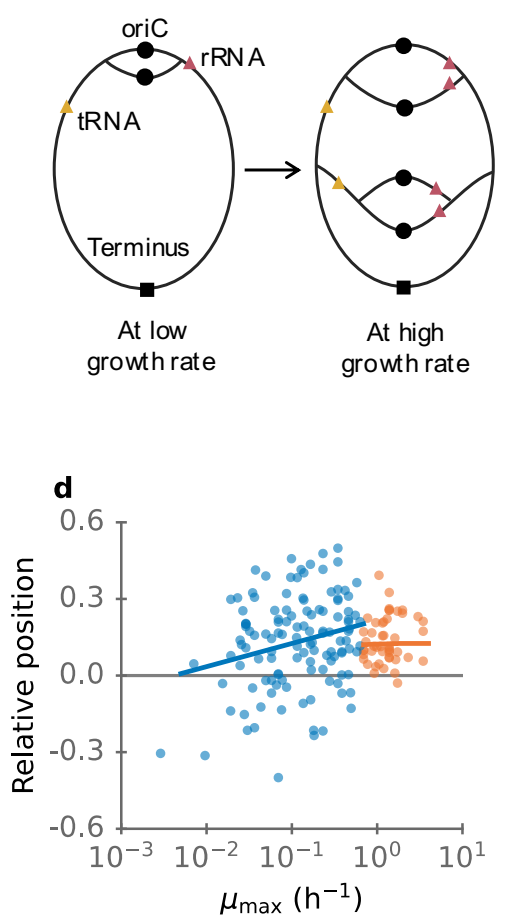
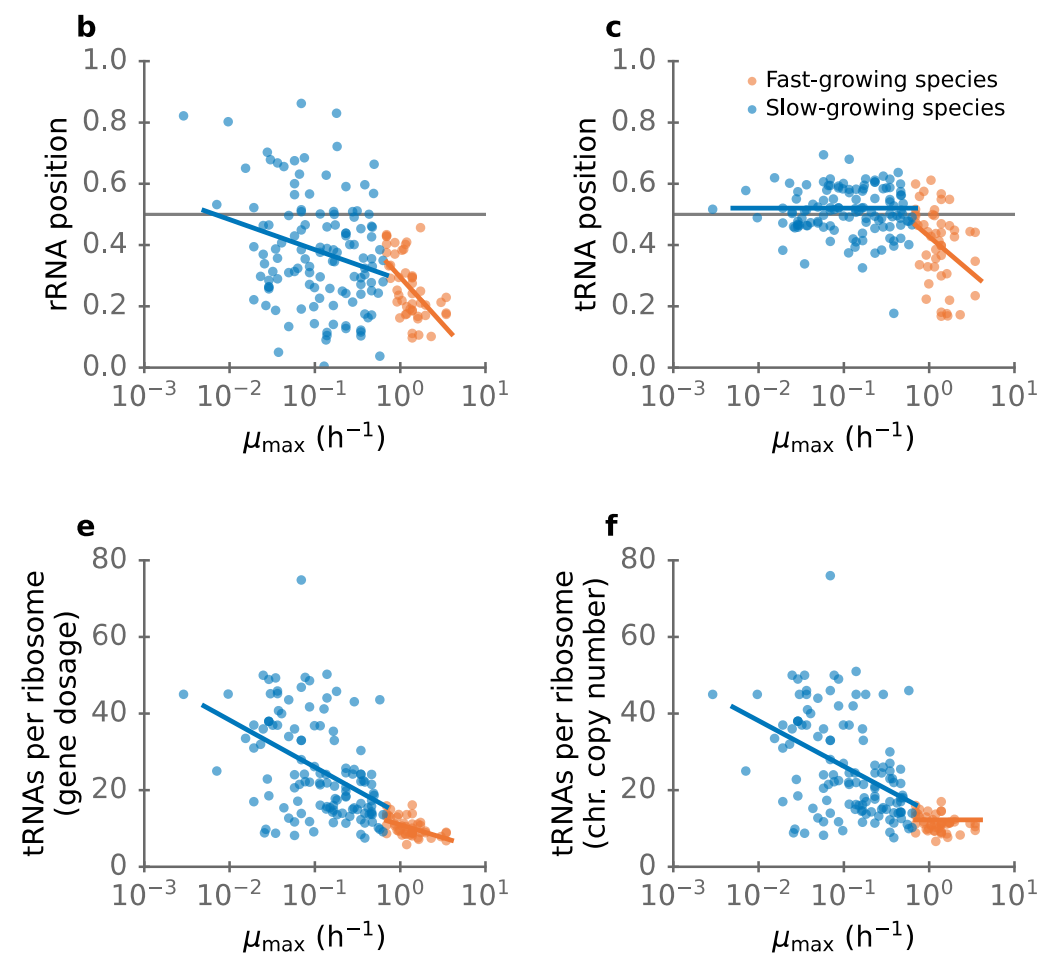

Figure 2. The genomic positioning of tRNA and rRNA genes contributes to the regulation of

RNA composition. (a) A schematic diagram showing the dosage ratio of two genes as a function of growth rate, quantified in equations (3) and (16). (b) Arithmetic means of the tRNA positions for individual genomes as a function of $\mu_{\max }$. The horizontal grey line marks the midpoint between origin and terminus of replication. (c) Same for rRNA. (d) Relative positions between tRNA and rRNA genes (position ${ }_{\mathrm{tRNA}}-$ position $_{\mathrm{rRNA}}$ ). The horizontal grey line marks the relative position 0. (e) tRNA/rRNA dosage ratios. (f) Genomic tRNA/rRNA ratios. In (b)-(f), blue points indicate slow growing species (with blue linear regression line) and orange points indicate fast-growing species (with orange linear regression line). 
$\left(1 \mathrm{~h}^{-1} \leq \mu \leq 2 \mathrm{~h}^{-1}\right)$ is very close to the optimal prediction, which corresponds to about 9 tRNAs per ribosome. This result is consistent with the notion that at the highest growth rates, both tRNA and rRNA genes are transcribed at the maximally possible rate, such that their relative expression is indeed dominated by gene dosage effects. The expression of both tRNA and rRNA operons is regulated by the P1 promoter, which is repressed by ppGpp; at near-maximal growth rates, ppGpp concentrations are low and the P1 promoter works near its maximal capacity [53]. In contrast, at low growth rates, P1 is repressed by ppGpp, and thus gene dosage can only partially explain the tRNA/rRNA ratio in these conditions.

According to equation (1), faster growing species need a lower TC/ribosome ratio at maximal growth. We indeed find statistically highly significant negative correlations between the tRNA/ribosome ratio and $\mu_{\max }$ (Fig. 2e; slowly growing species: $\rho=-0.44, P=2.8 \times 10^{-7}$; fast-growing species: $\rho=-0.49, P=$ 0.00043). While slowly growing species show a wide range of tRNA/ribosome gene dosage ratios, the ratio in fast-growing species shows a much tighter distribution. The chromosomal tRNA/ribosome copy number ratios (Fig. 2f) in slowly growing species are almost identical to the gene dosage ratios.

150 Chromosomal gene copy number ratios in fast-growing species show a similarly tight distribution as gene dosage ratios (with $10^{\text {th }}$ percentile $=8.78$ and $90^{\text {th }}$ percentile $=14.5$ ), they show no strong systematic dependence on $\mu_{\max }$ (Fig. 2e, $\rho=-0.24, P=0.10$ ). Interestingly, we also find no statistically significant dependence of the relative position on $\mu_{\max }$ in fast-growing species (Fig. $\mathbf{2 d}, \rho=0.15, P=0.31$ ). Thus, the tRNA/ribosome ratio appears to be tightly constrained in fast-growing bacteria, and is likely regulated through replication-associated gene dosage effects in fast growth.

\section{Conclusions}

We conclude that the tRNA/ribosome ratio appears to be tightly constrained across fast-growing bacteria. Its regulation is likely dominated by replication-associated gene dosage effects in fast growth, implemented through the relative chromosomal positioning of tRNA and ribosomal RNA genes. The 
bioRxiv preprint doi: https://doi.org/10.1101/2021.02.05.429890; this version posted February 6, 2021. The copyright holder for this preprint (which was not certified by peer review) is the author/funder. All rights reserved. No reuse allowed without permission.

160 objective of this regulation is to not only increase the expression of TCs and ribosomes with growth rate, but to also adjust their relative concentrations according to the RNA composition growth law quantified by equations (1) and (2). 


\section{Methods}

\section{Derivation of the optimal TC/ribosome ratio}

165 In recent work, we have shown that the growth-rate dependent composition of the translation machinery in E. coli is accurately described by predictions based on detailed reaction kinetics and the numerical minimization of the total mass of all participating molecules [37]. This minimization was motivated by the observation that the cellular dry mass density is approximately constant across growth conditions [54]; the minimization of the energy consumed or the enzyme mass required for the production of the different molecules led to almost identical results [37].

Here, we consider a much simpler representation of the elongation step of protein translation, which can be modeled as an enzymatic reaction following Michaelis-Menten kinetics [5]. In this case, the minimization of the combined mass concentration of ribosome and TC can be performed analytically, as demonstrated by Dourado et al. [9]. At a given reaction flux $v$, the optimal concentration of the ribosome $R$ (the "enzyme") can be written as a function of $v$ [9]:

$$
[R]=\frac{v}{k_{c a t}}+\sqrt{\frac{K_{\mathrm{m}} \cdot v}{a \cdot k_{\mathrm{cat}}}} ;
$$

Here, $a$ is the ratio of molecular weights of enzyme and substrate, $k_{\text {cat }}$ is the turnover number of the ribosome, and $K_{\mathrm{m}}$ is the ribosome's Michaelis constant for TC. In E. coli, the molecular weight of the ribosome is $2307.0 \mathrm{kDa}$ and the molecular weight of a TC is $69.6 \mathrm{kDa}$ [37], thus $a=33.1$. For a single TC, $K_{\mathrm{m} \text {-singletc }}=3 \mu \mathrm{M}$ [5]; the effective number of TC [5] is 34 (the predicted expressed tRNA in Ref. [37]), and thus $K_{\mathrm{m}}=34 \cdot K_{\mathrm{m} \text {-singletc }}=102 \mu \mathrm{M}$. $k_{\mathrm{cat}}=22 \mathrm{~s}^{-1}$ is the observed maximal translation rate of a ribosome [5]. The optimal concentration of TCs (the "substrate" $M$ ) can also be expressed as a function of $v$,

$$
[\mathrm{TC}]=\sqrt{\frac{a \cdot K_{\mathrm{m}} \cdot v}{k_{\mathrm{cat}}}} .
$$

Thus, the TC/ribosome concentration ratio can be written as

$$
\frac{[\mathrm{TC}]}{[R]}=\frac{a \cdot \sqrt{k_{\mathrm{cat}} \cdot K_{\mathrm{m}}}}{\sqrt{a \cdot v}+\sqrt{k_{\mathrm{cat}} \cdot K_{\mathrm{m}}}} .
$$

At steady state, protein production rate $(v)$ equals to rate of protein dilution by volume growth,

$$
v=\mu \cdot P,
$$


with growth rate $\mu$ and total cellular protein concentration $P$ (in units of amino acids per volume). Thus, equation (6) can be rewritten as (equation (1) of the main text)

$$
\frac{[T C]}{[R]}=\frac{a \cdot \sqrt{k_{\mathrm{cat}} \cdot K_{\mathrm{m}}}}{\sqrt{a \cdot \mu \cdot P}+\sqrt{k_{\mathrm{cat}} \cdot K_{\mathrm{m}}}} .
$$

The protein concentration $P$ is calculated from E. coli proteome expression data [45] and cell volume [55] for growth on glucose,

$$
P=\frac{\sum_{i} N_{i} L_{i}}{V_{\text {cell }} N_{\mathrm{A}}},
$$

where $N_{i}$ is the copy number per cell and $L_{i}$ the length of protein $i$ [45], $V_{\text {cell }}$ is the cell volume [55], and $N_{\mathrm{A}}$ is the Avogadro constant. In a more recent publication [56], the authors of Ref. [55] re-measured the volume of cells by super-resolution microscopy and found that cell volume was overestimated in Ref. [55] (by a factor of $0.67^{-1}$ for growth on glucose). We thus modified cell volume by a factor of 0.67 relative to the values in Ref. [55], resulting in $P=1.16 \times 10^{6} \mu \mathrm{M}$.

By multiplying the left-hand side of equation (8) with the molecular weight ratio of tRNA to rRNA, we obtain the tRNA and rRNA mass ratio (equation (2) of the main text),

$$
\frac{M_{\mathrm{tRNA}}}{M_{\mathrm{rRNA}}}=\frac{[T C] \cdot m_{\mathrm{tRNA}}}{[R] \cdot m_{r R N A}}=r \cdot \frac{\sqrt{k_{\mathrm{cat}} \cdot K_{\mathrm{m}}}}{\sqrt{a \cdot \mu \cdot P}+\sqrt{k_{\mathrm{cat}} \cdot K_{\mathrm{m}}}}
$$

with

$$
r=a \cdot \frac{m_{\mathrm{tRNA}}}{m_{r R N A}}
$$

Here, $m_{\mathrm{tRNA}}$ is the molecular mass of tRNA, $m_{\mathrm{rRNA}}$ is the total mass of RNA in one ribosome, and $r$ is the ratio of the tRNA mass fraction of a TC and the rRNA mass fraction of the ribosome. For bacteria, we use data from $E$. coli $\left(m_{\mathrm{tRNA}}=25.8 \mathrm{kDa}, m_{\mathrm{rRNA}}=1480 \mathrm{kDa}\right)$, resulting in $a=33.1$ and $r=0.58$. For eukaryotes, we use data from S. cerevisiae, resulting in $a=40.3$ and $r=0.59$; the molecular weights of the ribosome (3044.4 kDa), rRNA (1750 kDa), TC (75.6 kDa), and tRNA (25.6 kDa) were calculated from the respective sequences according to the Saccharomyces Genome Database [57].

\section{Gene positions}

The chromosomal position of the center of the origin of replication (oriC) for different genomes was obtained from the DoriC database (version 10.0) [58]. The start and end positions of rRNA and tRNA 
genes were downloaded from the RefSeq database (Release 93, downloaded on April 09, 2019); gene locations were defined as the midpoint between gene start and end. We defined gene position as the relative distance of a gene to oriC, calculated as the shortest distance between the gene and oriC on the circular chromosome, divided by half the length of the chromosome. Gene position ranges from 0 to 1.

210 For some species, more than one oriC has been annotated [58]. However, we found that all oriCs are very close on the chromosome in these species: the maximal distance between two oriCs is less than $1 \%$ of the chromosome length. Thus, different oriCs are expected to have a negligible effect on gene position and we randomly selected one of the oriCs to calculate gene position.

\section{Maximal growth rate dataset}

215 Minimal doubling times $\tau_{\min }$ (in hours) were obtained from Ref. [49] and were converted to maximal growth rates as $\mu_{\max }=\frac{\ln (2)}{\tau_{\min }}$. For the analyses, we only used species for which we additionally had genome annotation and oriC location, and which had only one chromosome. The final trimmed dataset contains 170 species (Additional file 1: Table S3).

\section{Gene dosage}

220 We used the Cooper-Helmstetter model $[29,30]$ to calculate gene dosage. The model is briefly summarized below. Let $C$ be the time required to replicate the chromosome; let $D$ be the time between the termination of a round of replication and the next cell division; let $\tau$ be the doubling time. The dosage of gene $i\left(X_{i}\right)$ is then given by:

$$
X_{i}=2^{\frac{C\left(1-\text { position }_{i}\right)+D}{\tau}}
$$

Where position $_{i}$ is the genomic position of gene $i$. With

$$
\begin{gathered}
\tau=\frac{\ln (2)}{\mu}, \\
X_{i}=e^{\mu\left[C\left(1-\text { position }_{i}\right)+D\right]}
\end{gathered}
$$

The gene dosage ratio of two genes $\left(X_{i} / X_{j}\right)$ is then (equation (3) of the main text)

$$
\frac{X_{i}}{X_{j}}=e^{\mu C\left(\text { position }_{j}-\text { position }_{i}\right)} .
$$


Each genome contains multiple tRNA and rRNA genes. In this case, we use the ratio of the total gene dosages,

$$
\frac{\sum X_{t R N A}}{\sum X_{\text {ribosome }}}=\frac{\sum X_{t R N A}}{\frac{1}{n} \sum X_{r R N A}}=\frac{\sum e^{\mu\left[C\left(1-\text { position }_{t R N A}\right)+D\right]}}{\frac{1}{n} \sum e^{\mu\left[C\left(1-\text { position }_{r R N A}\right)+D\right]}}=\frac{\sum e^{\mu C\left(1-\text { position }_{t R N A}\right)}}{\frac{1}{n} \sum e^{\mu C\left(1-\text { position }_{r R N A}\right)}}
$$

where $n=3$ is the number of rRNA genes per ribosome.

We assumed a constant DNA replication rate of $k_{\text {rep }}=1000 \mathrm{bp} \mathrm{s}^{-1}$ [27] to calculate the C-period as

$$
C=\frac{L_{\text {genome }}}{2 k_{\text {rep }}}
$$

with $L_{\text {genome }}$ the length of the given genome. 


\section{Supplementary information}

\section{Additional file 1: Supplementary Tables}

Table S1. Ternary complex per ribosome in E. coli.

Table S2. tRNA per rRNA in other species.

Table S3. The maximal growth rate dataset, including tRNA and rRNA positions, copies, and dosages.

\section{Declarations}

240 Ethics approval and consent to participate

Not applicable.

\section{Consent for publication}

Not applicable.

\section{Availability of data and materials}

245 The experimental data used in this study and the corresponding original publications is provided in Additional file 1.

\section{Competing interests}

The authors declare that they have no competing interests.

\section{Funding}

250 This work was supported by the Volkswagen Foundation under the "Life?" initiative, and by the German Research Foundation (DFG) through grant CRC 1310, and, under Germany's Excellence Strategy, through grant EXC 2048/1 (Project ID: 390686111).

\section{Acknowledgments}

We thank Hugo Dourado, Peter Schubert, and Deniz Sezer for helpful discussions.

\section{Author Contributions}

$\mathrm{XPH}$ designed the study and performed the analyses. XPH and MJL interpreted the results and wrote the manuscript. 


\section{References}

1. Schaechter M, Maaløe O, Kjeldgaard NO. Dependency on medium and temperature of cell size and chemical composition during balanced growth of Salmonella typhimurium. J Gen Microbiol. 1958;19:592-606.

2. Bremer H, Dennis PP. Modulation of chemical composition and other parameters of the cell at different exponential growth rates. EcoSal Plus. 2008;3:765-77.

3. Scott M, Gunderson CW, Mateescu EM, Zhang Z, Hwa T. Interdependence of cell growth and gene expression: origins and consequences. Science. 2010;330:1099-102.

4. You C, Okano H, Hui S, Zhang Z, Kim M, Gunderson CW, et al. Coordination of bacterial proteome with metabolism by cyclic AMP signalling. Nature. 2013;500:301-6.

5. Klumpp S, Scott M, Pedersen S, Hwa T. Molecular crowding limits translation and cell growth. Proc Natl Acad Sci U S A. 2013;110:16754-9.

270 6. Dai X, Zhu M, Warren M, Balakrishnan R, Patsalo V, Okano H, et al. Reduction of translating ribosomes enables Escherichia coli to maintain elongation rates during slow growth. Nat Microbiol. 2016;2:16231.

7. Erickson DW, Schink SJ, Patsalo V, Williamson JR, Gerland U, Hwa T. A global resource allocation strategy governs growth transition kinetics of Escherichia coli. Nature. 2017;551:119-23.

8. Mori M, Schink S, Erickson DW, Gerland U, Hwa T. Quantifying the benefit of a proteome reserve in 275 fluctuating environments. Nat Commun. 2017;8:1-8.

9. Dourado H, Maurino V, Lercher M. Enzymes and substrates are balanced at minimal combined mass concentration in vivo. bioRxiv. 2017;doi: https://doi.org/10.1101/128009.

10. O'Brien EJ, Lerman J a, Chang RL, Hyduke DR, Palsson B $\varnothing$. Genome-scale models of metabolism and gene expression extend and refine growth phenotype prediction. Mol Syst Biol. 2013;9:693.

280 11. Bosdriesz E, Molenaar D, Teusink B, Bruggeman FJ. How fast-growing bacteria robustly tune their ribosome concentration to approximate growth-rate maximization. FEBS J. 2015;282:2029-44.

12. Kjeldgaard NO, Kurland CG. The distribution of soluble and ribosomal RNA as a function of growth rate. J Mol Biol. 1963;6:341-8.

13. Doi RH, Igarashi RT. Relation of ribonucleic acid composition to growth rate and dormancy in Bacillus 285 subtilis. Nature. 1964;203:1092-4.

14. Panos C, Hynes LM, Cohen M. On RNA composition and growth rates of A streptococcus and derived stable L-form. Biochem Biophys Res Commun. 1965;19:62-6.

15. Rosset R, Julien J, Monier R. Ribonucleic acid composition of bacteria as a function of growth rate. J Mol Biol. 1966;18:308-20.

290 16. Sykes J, Young TW. Studies on the ribosomes and ribonucleic acids of Aerobacter aerogenes grown at different rates in carbon-limited continuous culture. BBA Sect Nucleic Acids Protein Synth. 1968;169:103-16.

17. Alberghina FAM, Sturani E, Gohlke JR. Levels and rates of synthesis of ribosomal ribonucleic acid, transfer ribonucleic acid, and protein in Neurospora crassa in different steady states of growth. J Biol 
Chem. 1975;250:4381-8.

18. Waldron C, Lacroute F. Effect of growth rate on the amounts of ribosomal and transfer ribonucleic acids in yeast. J Bacteriol. 1975;122:855-65.

19. Furano A V. Content of elongation factor Tu in Escherichia coli. Proc Natl Acad Sci. 1975;72:4780-4.

20. Dong H, Nilsson L, Kurland CG. Co-variation of tRNA abundance and codon usage in Escherichia coli at different growth rates. J Mol Biol. 1996;260:649-63.

21. Potrykus K, Murphy H, Philippe N, Cashel M. ppGpp is the major source of growth rate control in $E$. coli. Environ Microbiol. 2011;13:563-75.

22. Zhu M, Dai X. Growth suppression by altered ( $p$ )ppGpp levels results from non-optimal resource allocation in Escherichia coli. Nucleic Acids Res. 2019;47:4684-93.

305 23. Towbin BD, Korem Y, Bren A, Doron S, Sorek R, Alon U. Optimality and sub-optimality in a bacterial growth law. Nat Commun. 2017;8:1-8.

24. Rocha EPC. The Organization of the Bacterial Genome. Annu Rev Genet. 2008;42:211-33.

25. Pang TY, Lercher MJ. Supra-operonic clusters of functionally related genes (SOCs) are a source of horizontal gene co-transfers. Sci Rep. 2017;7:1-10.

310 26. Gao N, Lu G, Lercher MJ, Chen WH. Selection for energy efficiency drives strand-biased gene distribution in prokaryotes. Sci Rep. 2017;7:1-10.

27. Couturier E, Rocha EPC. Replication-associated gene dosage effects shape the genomes of fastgrowing bacteria but only for transcription and translation genes. Mol Microbiol. 2006;59:1506-18.

28. Yoshikawa H, O'Sullivan A, Sueoka N. Sequential replication of the Bacillus subtilis chromosome, III. 315 Regulation of initiation. Proc Natl Acad Sci. 1964;52:973-80.

29. Cooper S, Helmstetter CE. Chromosome replication and the division cycle of Escherichia coli B/r. J Mol Biol. 1968;31:519-40.

30. Bremer $\mathrm{H}$, Churchward $\mathrm{G}$. An examination of the Cooper-Helmstetter theory of DNA replication in bacteria and its underlying assumptions. J Theor Biol. 1977;69:645-54.

320 31. Kothapalli S, Nair S, Alokam S, Pang T, Khakhria R, Woodward D, et al. Diversity of genome structure in Salmonella enterica serovar Typhi populations. J Bacteriol. 2005;187:2638-50.

32. Campo N, Dias MJ, Daveran-Mingot M-L, Ritzenthaler P, Le Bourgeois P. Chromosomal constraints in Gram-positive bacteria revealed by artificial inversions. Mol Microbiol. 2004;51:511-22.

33. Hill CW, Gray JA. Effects of chromosomal inversion on cell fitness in Escherichia coli K-12. Genetics. 325 1988;119:771-8.

34. Louarn JM, Bouché JP, Legendre F, Louarn J, Patte J. Characterization and properties of very large inversions of the E. coli chromosome along the origin-to-terminus axis. Mol Gen Genet. 1985;201:46776.

35. Soler-Bistué A, Timmermans M, Mazel D. The proximity of ribosomal protein genes to oric enhances Vibrio cholerae fitness in the absence of multifork replication. Parkhill J, editor. MBio. 2017;8. 
36. Dourado H, Lercher MJ. An analytical theory of balanced cellular growth. Nat Commun. 2020;11:1226.

37. Hu X-P, Dourado H, Schubert $P$, Lercher MJ. The protein translation machinery is expressed for maximal efficiency in Escherichia coli. Nat Commun. 2020;11:5260.

335 38. Condon C, Philips J, Fu ZY, Squires C, Squires CL. Comparison of the expression of the seven ribosomal RNA operons in Escherichia coli. EMBO J. 1992;11:4175-85.

39. Ardell DH, Kirsebom LA. The genomic pattern of tDNA operon expression in E. coli. PLoS Comput Biol. 2005;1:e12.

40. Russell JB, Cook GM. Energetics of bacterial growth: balance of anabolic and catabolic reactions. Microbiol Rev. 1995;59:48-62.

41. Wong F, Dutta A, Chowdhury D, Gunawardena J. Structural conditions on complex networks for the Michaelis-Menten input-output response. Proc Natl Acad Sci. 2018;115:9738-43.

42. Milo R. What is the total number of protein molecules per cell volume? A call to rethink some published values. Bioessays. 2013;35:1050-5.

345 43. Mori T, Kishimoto S, Ijiro K, Kobayashi A, Okahata Y. Stoichiometric growth model for riboflavinproducing Bacillus subtilis. Biotechnol Bioeng. 2001;76:132-43.

44. Hanegraaf PPF, Muller EB. The dynamics of the macromolecular composition of biomass. J Theor Biol. 2001;212:237-51.

45. Schmidt A, Kochanowski K, Vedelaar S, Ahrne E, Volkmer B, Callipo L, et al. The quantitative and condition-dependent Escherichia coli proteome. Nat Biotechnol. 2016;34:104-10.

46. Skjold AC, Juarez $\mathrm{H}$, Hedgcoth C. Relationships among deoxyribonucleic acid, ribonucleic acid, and specific transfer ribonucleic acids in Escherichia coli 15T- at various growth rates. J Bacteriol. 1973;115:177-87.

47. Forchhammer J, Lindahl L. Growth rate of polypeptide chains as a function of the cell growth rate in a mutant of Escherichia coli 15. J Mol Biol. 1971;55:563-8.

48. Mann N, Carr NG. A constant ratio of transfer to ribosomal ribonucleic acid in Anacystis nidulans grown with differing mean generation times. Biochem Soc Trans. 1973;1:702-4.

49. Vieira-Silva S, Rocha EPC. The systemic imprint of growth and its uses in ecological (meta)genomics. PLoS Genet. 2010;6.

360 50. Rocha EPC. The replication-related organization of bacterial genomes. Microbiology. 2004;150:160927.

51. Michelsen O, Teixeira de Mattos MJ, Jensen PR, Hansen FG. Precise determinations of C and D periods by flow cytometry in Escherichia coli K-12 and B/r. Microbiology. 2003;149:1001-10.

52. Bipatnath $\mathrm{M}$, Dennis PP, Bremer H. Initiation and velocity of chromosome replication in Escherichia coli B/r and K-12. J Bacteriol. 1998;180:265-73.

53. Gourse RL, Gaal T, Bartlett MS, Appleman JA, Ross W. rRNA transcription and growth rate-dependent regulation of ribosome synthesis in Escherichia coli. Annu Rev Microbiol. 1996;50:645-77. 
54. Kubitschek HE, Baldwin WW, Schroeter SJ, Graetzer R. Independence of buoyant cell density and growth rate in Escherichia coli. J Bacteriol. 1984;158:296-9.

370 55. Volkmer B, Heinemann M. Condition-dependent cell volume and concentration of Escherichia coli to facilitate data conversion for systems biology modeling. PLoS One. 2011;6:1-6.

56. Radzikowski JL, Vedelaar S, Siegel D, Ortega ÁD, Schmidt A, Heinemann M. Bacterial persistence is an active $\sigma^{S}$ stress response to metabolic flux limitation. Mol Syst Biol. 2016;12:882.

57. Cherry JM, Hong EL, Amundsen C, Balakrishnan R, Binkley G, Chan ET, et al. Saccharomyces Genome Database: the genomics resource of budding yeast. Nucleic Acids Res. 2012;40:D700-5.

58. Luo H, Gao F. DoriC 10.0: an updated database of replication origins in prokaryotic genomes including chromosomes and plasmids. Nucleic Acids Res. 2019;47:D74-7. 